\title{
Questioning the Value of the Graduate Record Examinations (GRE) in PhD Admissions in Biomedical Engineering
}

\author{
(Published online 22 June 2020)
}

An increasing number of $\mathrm{PhD}$ programs across science, technology, engineering, and mathematics (STEM) fields have decided to drop their GRE requirement in $\mathrm{PhD}$ admissions, a trend that has been dubbed "GRExit" after the Brexit referendum in the UK. In the biological sciences, the use of the GRE as a measure of ability in biological research has been viewed skeptically by admissions committees for some time. However, since the GRE had been in use for many years the criticism mostly amounted to anecdotal reports and no systematic analysis was undertaken until the early 1990s when several national agencies were beginning to support the participation of Under Represented (UR) students in biomedical research. There was concern that the GRE was unfairly excluding UR students from being correctly evaluated in terms of their abilities to perform high level research. This led us at Vanderbilt to collect detailed data on admissions and outcomes of all graduate students in this area. We recorded scores from the GRE general test (Verbal and Quantitative). Subsequently we recorded the likelihood of completing the $\mathrm{PhD}$ degree (attrition) as well as the time to degree completion and productivity in terms of journal articles written as well as reviews and talks presented.

For a time, beginning in 2007 we recruited UR students independently of the applicant GRE scores for our umbrella biomedical research program. We still recorded incoming GRE scores but they were not used in admissions decisions. Subsequent analysis indicated that we had a wide range of such scores from our UR applicants [Moneta-Koehler L, et al., PLoS ONE 12:e0166742 (2017); Sealy L, et al., PLoS ONE 14:e0201634 (2019)]. But when these students graduated (at the same rate as majority students) they had an indistinguishable panel of overall performance measures. As it became well documented that there was a lack of any GRE evaluation effect, we continued UR recruitment as part of the regular admissions committee after 2011 until the GRE was no longer required 2 years ago.

During the period from 2000 onwards we corresponded with many comparable schools who were making the same determinations about the lack of any value in the GRE for predicting success in graduate school. Fully ten years before GRExit began it is fair to say that these observations were now being observed by many schools. Consequently after the first two Grexit papers were published there was a major commitment to dropping the use of GRE scores to determine who should be admitted to graduate school in the biomedical research environment. We now note that 300 departments and programs in the biomedical sciences have now accepted GRExit. As best we can determine, lacking GRE evaluations, good and creative research continues to be pursued in essentially all institutions which have taken this step. Evidently graduate school admissions committees are able to make sound decisions concerning the likely outcomes from the students they select. Almost certainly this reflects that the members of the admissions group are assaying a fairly wide range of parameters which their experience has taught them will lead to strong performance in research.

As we have observed no predictive value from GRE scores so far as performance in graduate school, equally important is the fact that even though UR students tend to perform in GRE tests with lower scores than majority students, they do equally as well in terms of overall outcomes in graduate school which depend upon creativity, dedication and commitment as well as intellectual productivity. We continue to believe that the decision we and many other institutions made to drop the GRE scores from our evaluation approaches for admission to graduate school was solidly based in data and has proved to be clearly the right decision.

In engineering, less data are publicly available to drive such analyses, yet historically we have also relied heavily on the GRE General Test and share many of the same concerns about equity, access and implicit bias. Since January 2018 one of us (M.R.K.) has served as the Chair Elect and current Chair of the BME Council of Chairs, an organization of 155 biomedical engineering departments in the U.S. and abroad. The Council of Chairs has been examining the GRExit issue, although plans for a large scale, data-driven study 
in BME was interrupted by the COVID-19 pandemic and the national crisis following the death of George Floyd. Interestingly, the challenges faced by college seniors due to the COVID-19 pandemic may prove to be the catalyst that encourages the majority of BME departments nationwide to conduct $\mathrm{PhD}$ admissions without consideration of GRE performance.

In June 2019, the BME Council of Chairs polled its members on whether they would be requiring the GRE General Test for Fall $2019 \mathrm{PhD}$ admissions. Of the departments who responded $(n=67), 88 \%$ indicated that they planned on requiring the GRE that fall. When the member departments were polled again on the GRE requirement in June $2020(n=42), 26 \%$ of the respondents indicated that they did not in fact require the GRE exam in Fall 2019, suggesting that more departments decided to drop this requirement between June and the fall application cycle of last year. Indeed, several programs proudly advertised their new "antiGRE" policy in booth displays at the 2019 Biomedical Engineering Society Annual Meeting in Philadelphia. The rest of the June 2020 poll results show an even more remarkable change. $17 \%$ of responding departments indicated that they will be dropping the GRE requirement for Fall 2020 and another 36\% are currently considering dropping the requirement, leaving only $21 \%$ of departmental respondents sure that they will continue to require the GRE exam. If half of the departments currently considering dropping the GRE follow through and do so, this would suggest that only $\sim 39 \%$ of departments nationally will require the GRE exam for $\mathrm{PhD}$ admissions this fall. It is likely that the current sea change in GRE policy within biomedical engineering is not completely motivated by the considerations of equity, access and systemic bias addressed in previous scholarly studies, but is an immediate reaction to alleviate the unique shelter-athome pandemic environment experienced by college seniors last semester. Nevertheless, it seems that due to the extraordinary once-in-a-generation circumstance of the COVID-19 pandemic, we may be embarking on a grand, national experiment on the impact of eliminating the GRE that may result in lasting change to the graduate admissions process in engineering.

Recently, the biomedical engineering faculty at Vanderbilt voted to eliminate the GRE requirement in
$\mathrm{PhD}$ admissions for the 2020-2021 academic year (fall and spring admissions), after which we will assess its impact in the admissions process. We are encouraged by the preliminary experiences of our colleagues in chemical and biomolecular engineering, who made the change to eliminate their GRE requirement in Fall 2019 for a 2-year trial.

The Chemical and Biomolecular Engineering Faculty made the decision to drop the GRE requirement based on the lack of correlation of GRE scores to the research performance of graduate students over the prior decade, as well as the desire to facilitate applications from a broader population of students by lowering the financial barrier. When evaluating applicants, the faculty placed enhanced scrutiny on letters, GPA, courses, past research performance, and interviews during campus visits. Even if the applicants had taken the GRE, their scores were not reported on the application or used in the evaluation. In general, the faculty did not seem to miss the GRE data in the evaluation process. In the first year of the trial as compared to the 2019 applicant pool, the number of graduate applications was slightly lower $(-3 \%)$, as was the percentage of U.S. URMs (7\% vs. $8 \%)$ and women $(36 \%$ vs. $42 \%)$. However, the percentage of applications from foreign nationals increased (53\% vs. $50 \%$ ), and in fact, students from Mexico and Central America were admitted and accepted offers to the program for the first time in over a decade. Importantly, the matriculating class consisted of $70 \%$ women and/or UR students, which is much greater than the range of $40-50 \%$ that the department has enrolled in recent years.

In the coming years we will see whether the graduate admission trends observed in the biomedical sciences prove to hold true for biomedical engineering, or whether there might be something inherently different within engineering that precludes the complete elimination of the GRE in PhD admissions, such as the need for expanded quantitative skills, or greater reliance on international applicants. In any case, we may all agree that the desire to make admissions more equitable, diverse, and inclusive is a worthy goal, and that a generally more holistic review of application materials is the best approach to achieve this. As the recent grassroots movements tagged as 
\#ShutDownAcademia, \#ShutDownSTEM, and \#Strike4BlackLives suggest (see, for instance, shutdownstem.com), UR academics and their allies strongly feel that the time has come to reexamine our biases and make positive change.

Michael R. King

Department of Biomedical Engineering

Vanderbilt University

Electronic mail: mike.king@vanderbilt.edu

G. Kane Jennings

Department of Chemical and Biomolecular

Engineering, Vanderbilt University, Nashville, TN,

USA
Roger G. Chalkley and Linda J. Sealy

Department of Molecular Physiology \& Biophysics, Vanderbilt University, Nashville, TN, USA

Publisher's Note Springer Nature remains neutral with regard to jurisdictional claims in published maps and institutional affiliations. 